\title{
An Extended Kalman Filter for In Situ Sensing of Yttria-Stabilized Zirconia in Chemical Vapor Deposition
}

\author{
Rentian Xiong, Paul J. Wissmann, and Martha A. Gallivan \\ School of Chemical \& Biomolecular Engineering \\ Georgia Institute of Technology \\ Atlanta, GA 30332-0100, USA
}

\begin{abstract}
Chemical vapor deposition (CVD) is an important step in integrated circuit fabrication and is a dynamic process that is complex and subject to unmeasured disturbances. Currently in semiconductor industry the main form of advanced process control for CVD is run-to-run (R2R) control. $R 2 R$ control is a form of discrete process control in which the processing recipe is modified ex situ based on post-process metrology data. Ideally one would like to monitor and control film growth in situ during deposition. To achieve this goal an in situ sensor that can sense film properties in real time is needed. This paper studied an extended Kalman filter (EKF) based in situ sensor and demonstrated its application in chemical vapor deposition of yttria stabilized zirconia (YSZ). The sensor consists of a reflectometer, a data preprocessing module, and an EKF algorithm. The reflectometer measures reflected light intensity from the film surface at two wavelengths, $950 \mathrm{~nm}$ and $470 \mathrm{~nm}$. The data preprocessing module calibrates raw intensity data, estimates the initial state and triggers EKF. EKF estimates film thickness, roughness, and growth rate by minimizing the mismatch between the estimated and measured reflectance. The estimated film properties were compared with ex situ characterization using scanning electron microscopy (SEM) and atomic force microscopy (AFM). Sensitivity and robustness of EKF were also studied by both simulation and experimental implementation.
\end{abstract}

Keywords: In-situ sensing, film growth, chemical vapor deposition, extended Kalman filter, roughness, soft sensor

\section{INTRODUCTION}

Chemical vapor deposition (CVD) is a widely used method to deposit crystalline and amorphous thin films on solid surfaces [1]. The deposition of material on a solid surface is used to produce a wide range of industrially important materials and systems such as ultra-hard mechanical coatings, fuel cells, and solar cells [2], [3], [4], [5]. CVD is particularly important in the production of semiconductors and related electronic components. It is by far the most important area of CVD and is estimated to comprise threequarters of all CVD production [6].

CVD is a dynamic process that is complex and subject to unmeasured disturbances. It also exhibits steady drift in performance over time because of the contaminants and build-up of material inside the deposition chamber. To compensate for the process variability, improve yield and reduce cost, significant research efforts have been made to

To who correspondence should be addressed: E-mail: martha.gallivan@chbe.gatech.edu develop automatic control for CVD and other processes in microelectronics manufacturing over the past twenty years. The early stage of process control is mainly in the form of statistical process control (SPC). SPC is a technique that monitors process output in order to detect variations in the process. Variations are usually identified by applying a set of rules such as the Western Electric Company Rules to the output data. SPC is useful to monitor a process's stability and consistency, but it does not provide control actions that are necessary to correct variations [7]. To solve this problem, run to run control $(R 2 R)$ was developed in the 1990 s and currently it is the main form of advanced process control implemented in industry. R2R control is a type of discrete process control in which the processing recipe is modified ex situ, i.e., between CVD runs, based on postprocess ex situ metrology data. In practice a R2R controller acts as a supervisor for the real-time equipment controller. Based on post-process measurement, the R2R controller decides whether a recipe change is needed and suggests a new recipe for use in the next run. The recipe consists of the regulatory set points for manipulated variables and is downloaded to an equipment controller which applies feedback loops to execute the recipe [8]. The problem with $\mathrm{R} 2 \mathrm{R}$ control lies in its discrete nature, i.e. control actions only take place between runs and thus it can not correct the problems that happened in one batch. Ideally one would like to monitor and control film properties in situ during deposition. Recent work in this direction includes in situ control of film roughness in simulation using kinetic Monte Carlo simulations and nonlinear PDEs [9], [10]. Optical measurements of film properties have been demonstrated for control of thickness and composition [11], [12] in deposition and etching, but implementation of in-situ control has been limited due to the lack of in situ sensing capability in practice [7]. In fact in situ sensing has always been a primary limitation for controller development in semiconductor industry. This is because most semiconductor manufacturing environments involve high temperature, high vacuum and corrosive materials that make in situ sensor implementation difficult. In the International Technology Roadmap for Semiconductors 2003 edition, in situ sensing was identified as one of the grand challenges in the near term (through 2009).

Previous research on in situ sensing has been focused on using in situ characterization tools to study the reaction 
mechanism and monitor film growth. For example in situ mass spectroscopy was used to identify the reactant species and their concentration in gas phase during deposition [13], [14]. In situ FTIR and Raman spectroscopy were used to determine the composition of the deposited film and byproduct [15], [16]. Other tools like in situ X-ray diffraction (XRD) and X-ray photoelectrons spectrometry (XPS) have also been reported to identify the crystal structure and composition of the film [17], [18], [19]. These tools, however, are not suitable for the routine in situ sensing and control in CVD because they are expensive, slow, and difficult to operate. To solve this problem, some optical sensors were developed and these include thermal emission, emission reflectance, and ellipsometry [20], [21], [11], [22]. Optical sensors are a natural choice for in situ sensing because they are nonperturbing, sensitive, and can be installed outside the deposition chamber. Optical sensors are also cheap, commercially available, and easy to operate. Among optical sensors laser reflectometry (LR) and spectroscopic ellipsometry (SE) are the most popular [23], [24], [25], [26].

Optical sensors do not measure film properties (e.g. thickness, roughness) directly. Instead they measure the state change of the probe light after it interacts with the film. For example, spectroscopic ellipsometry measures the change of the polarization state of the probe light caused by the film, which is a function of film characteristics. Laser reflectometry measures specular reflectance change due to interference as the film grows. Film properties must be extracted through a model based analysis using optical physics. For example, in laser reflectometry a thin film interference model was used to relate film thickness to reflectance oscillations. Each of the interference peaks corresponds to a film thickness of half the wavelength of the light. Therefore by counting the number of interference peaks film thickness could be estimated [27], [28]. The peak counting method is extremely easy to use but it requires multiple interference peaks to process. But in some cases depending on the film thickness desired and the wavelength of the light, there may be only a few oscillations during the entire film growth. Besides, the peak counting method cannot estimate film thickness between interference peaks and thus it is not a real-time method in the strict sense. A more rigorous approach is the least square fitting method. In this method a sensor model is first developed, and then measurements are fitted into the model to extract parameters like thickness and film optical constants. This method can process measurements between interference peaks but it is very sensitive to the initial guess of film state. This is because the least square fit error surface may contain several local minima. Besides depending on the number of states to be estimated, multiple measurements have to be obtained. For example, Breiland used a virtual interface model to extract three parameters (growth rate and complex refractive index of GaAs film) plus two fitting parameters which represented the cumulative optical response of all the underlying virtual layers [29]. At least five measure- ments were needed to get a least square solution. The film properties and growth rate between the five measurements were assumed to be constant. The sensor models based on physical optics are usually complicated and in many cases several assumptions were made to simplify the model. For example surface roughness was usually discarded. However, surface roughness could have multiple effects on optical measurements such as emissivity of the surface and normal reflectance by scattering the light [30]. By neglecting the roughness one loses information contained in the raw measurement data, and introduces errors into the estimates of other properties.

The Kalman filter represents another approach to extract film properties from indirect measurements. A Kalman filter is an optimal state estimator applied to a dynamic system that involves random perturbations. It gives a linear, unbiased, and minimum error variance recursive algorithm to optimally estimate the unknown state of a linear dynamic system from noisy data taken at discrete times [31], [32]. For a nonlinear system, a linearization has to be made at each local estimated state and has proven to be useful in many applications. Compared to peak counting and least square fitting methods, Kalman filter requires only the most recent measurement to update the state. Therefore it is more computationally efficient and ideal for in-situ sensing and feedback control. Kalman filters have been widely used in many areas of industrial applications such as video and laser tracking systems, satellite navigation, etc. [31]. There have also been a few applications of Kalman filtering in CVD and other surface processing. An early application of Kalman filtering to in-situ monitoring of a deposition process appeared in 1984 [33]. Later Woo and his coworkers applied a modified Kalman filter to estimate film thickness and growth rate from a laser reflectance measurement [34]. EKF was also used to estimate etch rate and end-point in a plasma etch system by Vincent and Khargonekar [12], [35]. In both their works, adjustable parameters called forgetting factor in Woo and optical gain in Vincent have to be included to account for the deficiency of the sensor model. However, in many cases film roughness and changing extinction coefficient caused the reflected light amplitude to decay. By using adjustable parameters, the information contained in the amplitude change was lost.

This study used a more realistic sensor model that accounts for the reflectance amplitude decay due to surface roughness and film absorption. A preprocessing module was used to calibrate the raw reflected light intensity data so that there is no need for adjustable scaling parameters. The EKF was capable of adaptive system identification so that little a priori knowledge of process dynamics is required. The purpose of this study is to determine the amount of information that needs to be provided by the process model in order to simultaneously estimate film thickness and surface roughness from reflectance measurements. 


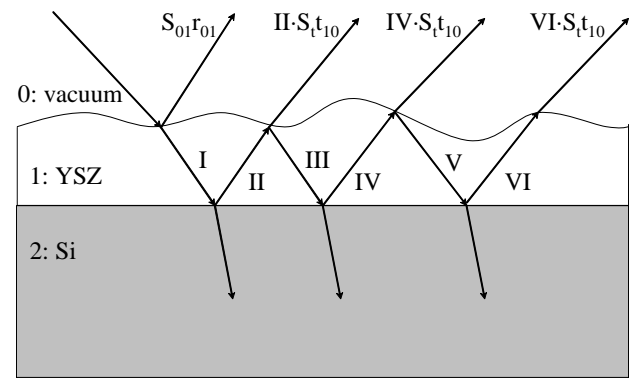

Fig. 1. Schematic diagram of the two layer model with rough surface for YSZ deposited on a silicon wafer.

\section{MODEL}

\section{A. Sensor model}

The performance of a Kalman filter is highly dependent upon the accuracy of the sensor model. There have been extensive research efforts on the modeling of light scattering on rough surfaces using various techniques such as ray tracing, Beckmann-Kirchhoff scattering model, and virtual interface model [36], [37]. The computational requirements of these models also vary. Most of these models deal with reflection from a single rough surface, but in our case the film interference effects also must be incorporated. In this paper the sensor model is based on the work of Filinski [38]. Yin et al. used this model to determine optical constants of diamond films with rough surfaces [39]. In that study the diamond film was free standing, so both sides of the film are in air. However, the derivation is easily modified to the case of a thin film deposited on a smooth silicon substrate. For the completeness of this paper, the derivation procedure is presented here. Figure 1 is the schematic diagram of the two layer model. It is well known that there is a native silicon oxide layer on top of a pure silicon wafer. The ellipsometry measurements of the silicon substrate used in this work indicated a native silicon oxide layer of around $1.6 \mathrm{~nm}$ was present. After heating up to deposition temperature (e.g. $700^{\circ} \mathrm{C}$ ) the thickness increased to $16.3 \mathrm{~nm}$ (under a vacuum of 1.5 torr). In either case the presence of thin oxide layers do not significantly affect YSZ film reflectance [40]. Therefore we could assume that no silicon oxide layer was present, which greatly simplified the derivation. Filinski's model started with Fresnel formulas which describe the amplitude of reflected and transmitted light when a beam of light is incident on an optically smooth surface, from medium 0 to medium 1. For normal incidence, the Fresnel formulas are

$$
\begin{aligned}
& r_{01}=\frac{\hat{n}_{1}-\hat{n}_{0}}{\hat{n}_{1}+\hat{n}_{0}} \\
& t_{01}=\frac{2 \hat{n}_{0}}{\hat{n}_{1}+\hat{n}_{0}}
\end{aligned}
$$

where $\hat{n}_{0}$ and $\hat{n}_{1}$ are the complex refractive index of medium 0 and 1 , respectively. When the surface is rough, part of the light is scattered and this causes the reflected intensity in the specular direction to decrease. In Filinski's model the amount of reduction was expressed in terms of scattering factors which depend on the root-mean-square (rms) surface roughness $\sigma$. The three scattering factors are expressed as follows:

$$
\begin{aligned}
S_{01} & =\exp \left[-\frac{1}{2}\left(\frac{4 \pi \operatorname{Re}\left(\hat{n}_{0}\right) \sigma}{\lambda}\right)^{2}\right] \\
S_{10} & =\exp \left[-\frac{1}{2}\left(\frac{4 \pi \operatorname{Re}\left(\hat{n}_{1}\right) \sigma}{\lambda}\right)^{2}\right] \\
S_{t} & =\exp \left[-\frac{1}{2}\left(\frac{2 \pi\left(\operatorname{Re}\left(\hat{n}_{1}\right)-\operatorname{Re}\left(\hat{n}_{0}\right)\right) \sigma}{\lambda}\right)^{2}\right]
\end{aligned}
$$

where $S_{01}$ is the scattering factor when light is incident from medium 0 to medium $1 . S_{10}$ is the scattering factor when light is incident from medium 1 to medium $0 . S_{t}$ is the scattering factor for transmitted light. $S_{t}$ does not change when incident direction changes. Using the above scattering factors, the intensity of the beams shown in Figure 1 can be expressed as:

$$
\begin{aligned}
I & =S_{t} \cdot t_{01} \\
I I & =I \cdot r_{12} \\
I I I & =I I \cdot S_{10} r_{10}=I \cdot r_{12} \cdot S_{10} r_{10} \\
I V & =I I I \cdot r_{12}=I \cdot r_{12}^{2} \cdot S_{10} r_{10} \\
V & =I V \cdot S_{10} r_{10}=I \cdot r_{12}^{2} \cdot\left(S_{10} r_{10}\right)^{2} \\
V I & =V \cdot r_{12}=I \cdot r_{12}^{3} \cdot\left(S_{10} r_{10}\right)^{2}
\end{aligned}
$$

Note that in the above equations, the interface between the YSZ film and the silicon substrate was assumed to be smooth. This assumption was proved to be appropriate as AFM scans of our silicon substrates indicated a roughness less than $10 \mathrm{~nm}$ over an area of $100 \times 100 \mu \mathrm{m}$. The total amplitude of reflected light is the summation of all individual reflected light. Multiple reflections in a thin film lead to an infinite series for transmitted and reflected light which is expressed as follows:

$$
\begin{aligned}
r= & S_{01} r_{01}+I I \cdot S_{t} t_{10} \cdot e^{-i 2 \delta}+ \\
& I V \cdot S_{t} t_{10} \cdot e^{-i 4 \delta}+V I \cdot S_{t} t_{10} \cdot e^{-i 6 \delta}+\ldots \\
= & S_{01} r_{01}+S_{t} t_{10} \cdot \\
& \left(I I \cdot e^{-i 2 \delta}+I V \cdot e^{-i 4 \delta}+V I \cdot e^{-i 6 \delta}+\ldots\right) \\
= & S_{01} r_{01}+I \cdot r_{12} \cdot S_{t} t_{10} \cdot e^{-i 2 \delta} \cdot \\
& {\left[1+r_{12} S_{10} r_{10} e^{-i 2 \delta}+\left(r_{12} S_{10} r_{10} e^{-i 2 \delta}\right)^{2}+\ldots\right] }
\end{aligned}
$$

where $e^{-i 2 \delta}, e^{-i 4 \delta}$, and $e^{-i 6 \delta}$ are the phase factors of different beams as they traveled different distance. The phase factor $\delta$ is a function of the average thickness of the film $d$, such that $\delta=2 \pi \hat{n}_{1} d / \lambda$. 
Using $\sum_{k=0}^{\infty} x^{k}=\frac{1}{1-x}$ for $x<1$, eq. (4) becomes

$$
\begin{aligned}
r= & S_{01} r_{01} \\
& +I \cdot r_{12} S_{t} t_{10} e^{-i 2 \delta} \cdot \frac{1}{1-r_{12} S_{10} r_{10} e^{-i 2 \delta}} \\
= & \frac{S_{01} r_{01}+S_{01} S_{10} r_{01}^{2} r_{12} e^{-i 2 \delta}+S_{t}^{2} t_{01} t_{10} r_{12} e^{-i 2 \delta}}{1+r_{01} r_{12} S_{10} e^{-i 2 \delta}}
\end{aligned}
$$

When the roughness is zero, the sensor model of eq. (5) reduces to a well known expression for the reflectance of a smooth thin film on a thick substrate. The virtual interface model used by Breiland and Killeen [29] is the special case of eq. (5) in which $\sigma=0 . r$ is a complex number containing phase information. It was used to calculate the normal reflectance through the following expression: $R=r^{*} \cdot r$, where $r^{*}$ is the complex conjugate of $r . R$ represents the percentage of the energy of incident light that was reflected back from the surface. The part of the reflected light that is detected by the reflectometer depend on the reactor geometry, relative position, and optical aperture of the photodiode sensor. Thus $R$ is proportional to the measured raw voltage signal.

\section{B. Process model}

The sensor model in eq. (5) indicates that film thickness will cause reflectance oscillations through the phase factor $e^{-i 2 \delta}$. Therefore when using a least square fitting method to invert the sensor model, it is possible to get multiple solutions depending on the initial guess. One distinction of the Kalman filter is that it can utilize information from various sources including a process dynamic model to yield a more balanced estimate. Incorporating a process dynamic model could help the Kalman filter to identify the direction of state evolution and thus help it jump out of the local minima on the error surface.

There have been some research efforts on the modeling of process dynamics of YSZ thin film growth. The dynamics of YSZ deposition fall into two regimes, the precursor diffusion controlled regime and the surface reaction controlled regime, depending on the deposition temperature. In the surface reaction controlled regime, Akiyama et al. obtained an Arrhenius-type equation to relate deposition rate with deposition temperature by using a simplified Monte Carlo simulation and then comparing to experimentally observed step coverage on micro-scale trenches [41].

Having a process model could improve the ability to estimate film properties. However in many cases, especially for a new material system, the dynamics of deposition are not well understood. In order to make the Kalman filter a general tool, a technique called Kalman filter with adaptive system identification was developed [42]. The basic idea is to treat some set parameters as a random constant vector and make it a new state variable so that it is optimally estimated in an adaptive way. The detailed algorithm is presented in next section. The simple process model used in this work is shown below,

$$
\begin{aligned}
h[j+1] & =h[j]+G[j] \Delta t \\
\sigma[j+1] & =\sigma[j]+w_{1}[j] \\
G[j+1] & =G[j]+w_{2}[j]
\end{aligned}
$$

where $h, \sigma$, and $G$ are film thickness, rms roughness, and film growth rate, respectively. $\sigma$ and $G$ were treated as random constant variables and are to be estimated adaptively. $h$ is simply the integration of $G$. By integrating $G$ over time, the process model could inform the Kalman filter that film thickness is changing monotonically and thus help identify direction of the estimate. $w_{1}$ and $w_{2}$ are are assumed to be uncorrelated zero mean Gaussian white noises. For correlated and colored systems, a noise modified Kalman filter could be applied but is not discussed in this study.

More complex process models could be used instead. For example, the real and imaginary parts of the film's refractive index affect the reflectance, and could also be estimated. However, given the simple process model and lack of coupling between states in the process model, the limited measurement data would not be sufficient to estimate both roughness and index of refraction. In fact, there is additional coupling in the process between the states (e.g. growth rate would affect roughness), which could be exploited to improve the observability properties of the system. At this time we do not have quantitatively accurate model to describe it. Instead, we use a qualitatively reasonable process model and a detailed sensor model, and investigate how the simple process model can aid in prediction.

\section{EXTENDED KALMAN FILTER}

A Kalman filter is the optimal linear estimator for a linear system [32], but our sensor model is a nonlinear function of the states in the process model. We applied the standard approach for the extended Kalman filter to our nonlinear system, in which the system is linearized about the current estimate at each point in time [31]. For the nonlinear system

$$
\begin{aligned}
\mathbf{x}_{k+1} & =\mathbf{f}_{k}\left(\mathbf{x}_{k}\right)+H_{k}\left(\mathbf{x}_{k}\right) \mathbf{w}_{k} \\
\mathbf{y}_{k} & =\mathbf{g}_{k}\left(\mathbf{x}_{k}\right)+\nu_{k}
\end{aligned}
$$

where $\mathbf{x}_{k}$ is the state at time $k$ and $\mathbf{y}_{k}$ is the measurement, and additive noise terms are included in the process $\left(\mathbf{w}_{k}\right)$ and measurement $\left(\nu_{k}\right)$. The extended Kalman filter equations are

$$
\begin{aligned}
P_{k, k-1}= & {\left[\frac{\partial \mathbf{f}_{k-1}}{\partial \mathbf{x}_{k-1}}\left(\hat{\mathbf{x}}_{k-1}\right)\right] P_{k-1, k-1}\left[\frac{\partial \mathbf{f}_{k-1}}{\partial \mathbf{x}_{k-1}}\left(\hat{\mathbf{x}}_{k-1}\right)\right]^{T} } \\
& +H_{k-1}\left(\hat{\mathbf{x}}_{k-1}\right) Q_{k-1} H_{k-1}^{T}\left(\hat{\mathbf{x}}_{k-1}\right) \\
\hat{\mathbf{x}}_{k \mid k-1}= & \mathbf{f}_{k-1}\left(\hat{\mathbf{x}}_{k-1}\right) \\
G_{k}= & P_{k, k-1}\left[\frac{\partial \mathbf{g}_{k}}{\partial \mathbf{x}_{k}}\left(\hat{\mathbf{x}}_{k \mid k-1}\right)\right]^{T} . \\
& {\left[\left[\frac{\partial \mathbf{g}_{k}}{\partial \mathbf{x}_{k}}\left(\hat{\mathbf{x}}_{k \mid k-1}\right)\right] P_{k, k-1}\left[\frac{\partial \mathbf{g}_{k}}{\partial \mathbf{x}_{k}}\left(\hat{\mathbf{x}}_{k \mid k-1}\right)\right]^{T}\right.}
\end{aligned}
$$




$$
\begin{aligned}
& \left.+R_{k}\right]^{-1} \\
P_{k, k}= & {\left[I-G_{k}\left[\frac{\partial \mathbf{g}_{k}}{\partial \mathbf{x}_{k}}\left(\hat{\mathbf{x}}_{k \mid k-1}\right)\right]\right] P_{k, k-1} } \\
\hat{\mathbf{x}}_{k \mid k}= & \hat{\mathbf{x}}_{k \mid k-1}+G_{k}\left(\mathbf{y}_{k}-\mathbf{g}_{k}\left(\hat{\mathbf{x}}_{k \mid k-1}\right)\right)
\end{aligned}
$$

where $f$ is our linear process model in eq. (6), and $g$ is our nonlinear sensor model in eq. (5). The Kalman gain at time $k$ is $G_{k}$, and the a priori and a posteriori error covariance matrices are $P_{k \mid k-1}$ and $P_{k \mid k}$, respectively. The estimate for the state at time $k$ is $\hat{\mathbf{x}}_{k}$. Because the EKF equations are recursive and algebraic, there is little computation required, making them ideal for real-time estimation and control.

In our reactor we have two reflectance measurements, at $470 \mathrm{~nm}$ and $950 \mathrm{~nm}$, so that the system has two measurements and three states. Due to the coupling of the growth rate and thickness states, the linearized system is observable, for any linearization of the sensor model, which can be established by checking that the observability matrix is full rank. If more wavelengths were available for measurement, then it might be possible to independently estimate the real or complex refractive indices of the film, but with two measurements we are limited to estimating film thickness and one other film property (roughness here).

The peak counting method of Zuiker [28] used one wavelength to estimate thickness, roughness, and absorption. This was only possible because three measurements at three adjacent extrema in the interference oscillations were used, and the growth rate, roughness, and absorption were assumed to remain constant over this entire interval. Thus, the problem reduced to the solving of three equations for three unknowns. The primary disadvantage of the method is that it provides infrequent estimates. Additionally, since it uses three measurements to compute three properties, it is extremely sensitive to noise.

\section{SIMULATION}

\section{A. Sensor model simulation}

The sensor model in eq. (5) indicates that film thickness, roughness and refractive index affect reflectance in different ways. Before applying EKF, it is beneficial to understand how these different film properties affect reflectance. By doing so we can get a general idea what information was contained in the measurement data and judge if the estimated film properties are physically realistic. The following simulation uses a wavelength of $470 \mathrm{~nm}$. The optical constants of silicon, YSZ, and yttria used in the simulations are from the literature [43], [44], [45].

Figure 2 shows the reflectance data simulated at three different film growth rate $(10,20$ and $40 \mathrm{~nm} / \mathrm{min})$ but with the zero surface roughness and extinction coefficient. As shown, a faster film growth rate gives a shorter oscillation period. Film thickness enters the sensor model through a phase factor $e^{-i 2 \delta}$. Because $\delta=2 \pi \hat{n}_{1} d / \lambda$, each oscillation corresponds to a thickness of half a wavelength in the film. This is the idea behind the peak counting method.

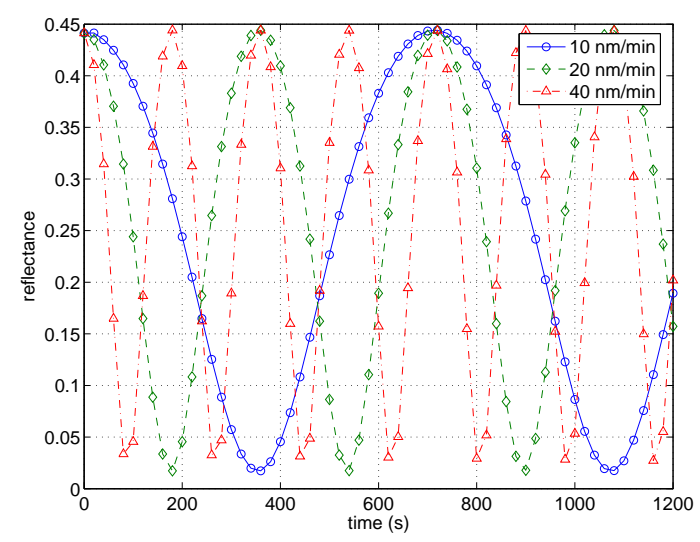

Fig. 2. Effect of film growth rate on reflectance.

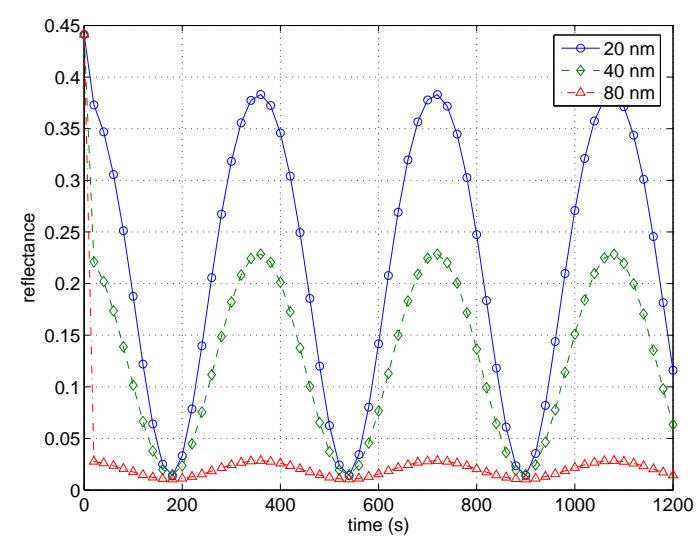

Fig. 3. Effect of constant surface roughness on reflectance.

Figure 3 shows the reflectance data simulated at three different rms roughnesses $(20,40$ and $50 \mathrm{~nm})$ but with the same film growth rate and extinction coefficient (imaginary part of the refractive index). As shown, a larger surface roughness causes more reduction in reflected light amplitude due to more energy loss in light scattering. However constant surface roughness does not cause the amplitude decay which was observed in many CVD experiments. Surface roughness enters sensor model through the three scattering factors. From the definition in eq. (2), the scattering factors are all real numbers and thus do not cause any phase change. Zuiker [28] used the following formula to calculate roughness.

$$
\sigma=\frac{1}{k} \sqrt{\frac{1}{2} \ln \left[\frac{2 r_{01}}{\sqrt{R_{\max }+R_{\min }}}\right]}
$$

where $k=2 \pi / \lambda$, and $R_{\max }$ and $R_{\min }$ are the peak and valley values of reflectance, respectively. This is consistent with Fig. 3, as overall amplitude reduction $\left(R_{\max }+R_{\min }\right)$ suggests a rougher surface.

In a real CVD process, the surface roughness would change with experimental and surface conditions. Luo et al. observed a reflectance increase in the first few minutes after deposition, after which it decayed gradually [46]. SEM 


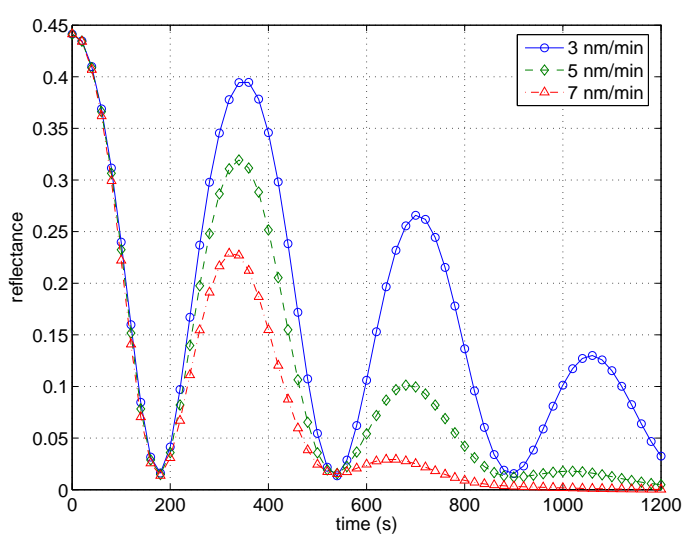

Fig. 4. Effect of increasing surface roughness on reflectance.

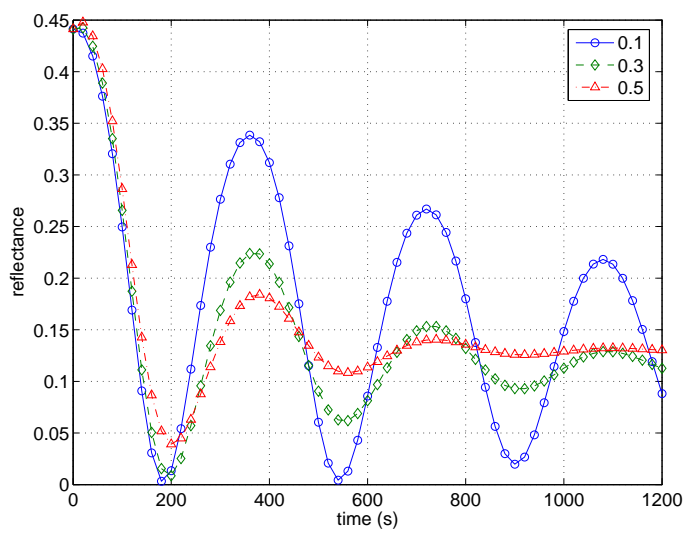

Fig. 5. Effect of extinction coefficient on reflectance.

pictures of the film suggested the increase of reflectance was caused by the coalescence of scattered polycrystalline grain islands to form a continuous film which had less roughness. As the film continued growing, the surface became rough again and reflectance decayed. The reflectance decay could be explained quite well by Figure 4, which shows simulated reflectance at different roughening rates (3 $\mathrm{nm} / \mathrm{min}, 5 \mathrm{~nm} / \mathrm{min}$ and $7 \mathrm{~nm} / \mathrm{min}$ ). Also note that increasing roughness caused the peak reflectance to decay much more than the valley reflectance.

Figure 5 shows the reflectance data simulated at three different extinction coefficients $(0.1,0.3$ and 0.5$)$ but with the same film growth rate and a smooth surface. As shown, film extinction coefficient also causes reflectance decay. But in contrast to roughness, it affects both peak and valley reflectance. Also light scattering due to roughness is a surface phenomenon, therefore for constant roughness, the film growth would not cause reflectance decay. However extinction coefficient is a film property, and for a constant extinction coefficient a thicker film would cause reflectance decay because the distance light traveled in the film increased. Also note that when thickness approaches infinity, according to the sensor model, the reflectance would approach $S_{01} r_{01}$ which is the reflectance on the air-
YSZ interface. This information was exploited by Breiland to extract the refractive index of a GaAs film [47].

\section{B. Extended Kalman filter simulations}

Before applying the EKF to real experimental reflectance data, we run simulations first in order to understand some characteristics of the filter, including robustness and sensitivity to tuning parameters. By doing so one could have a guideline in tuning EKF for real experimental data. EKF starts with four inputs: initial state estimate $\hat{\mathbf{x}}_{0}$, initial estimate covariance matrix $P_{0,0}$, covariance matrix of process model disturbance $Q_{k}$, and covariance matrix of sensor model noise $R_{k}$. The initial state estimate and its covariance matrix could be characterized ex-situ. For example one could measure initial film thickness using ellipsometry or SEM offline before CVD. Process model disturbances were difficult to model so in our implementation of EKF, $Q_{k}$ was used as a tuning parameter. Sensor noise, however, could be obtained from the histogram of the measurement. Therefore in the following simulation the effects of tuning parameters $Q_{k}$ and initial state estimate on the performance of EKF were studied. It was observed in many CVD experiments that film deposition rate was not constant throughout the reaction. This might be because the surface morphology (e.g. roughness, composition etc.) changed when a film was deposited on the substrate. Surface morphology then caused surface emissivity to change which determines heat radiation rate and surface temperature. Deposition rate is strongly dependent on deposition temperature. Therefore in this simulation we used an exponential decay function to simulate the film growth rate. Film thickness was then integrated over time. Surface roughness was also simulated using a linear function. It was observed that surface roughness increased during deposition and was attributed to textured growth in which slowly growing crystal faces are buried by faster growing faces. Extinction coefficients of YSZ were assumed to be random variables with mean value equal to that at room temperature. The standard deviation of sensor noise was chosen to be 0.001 which represents the noise level in our reflectometer. Figure 6 shows a typical simulated reflectance data. It has the main features of many reported reflectance measurements.

In the first simulation, EKF used the same initial state estimate as that used in data generation in order to investigate the effect of the turning parameter $Q_{k}$. For Case I, Figure 7 compares EKF estimated states and actual states. Figure 8 compares EKF generated reflectance from estimated states and model generated reflectance data as in Fig. 6. For Case II, simulation results were presented in Fig. 9 and 10. As shown, both $Q_{k}$ can give satisfactory estimates for film thickness. Surface roughness and film growth rate were not estimated exactly. However increasing $Q_{k}$ gives a better estimate for both surface roughness and film growth rate. It was clear from Fig. 9 that the trend of exponential decay of film growth rate and the linear increase of surface roughening rate were captured quite well. They were assumed 


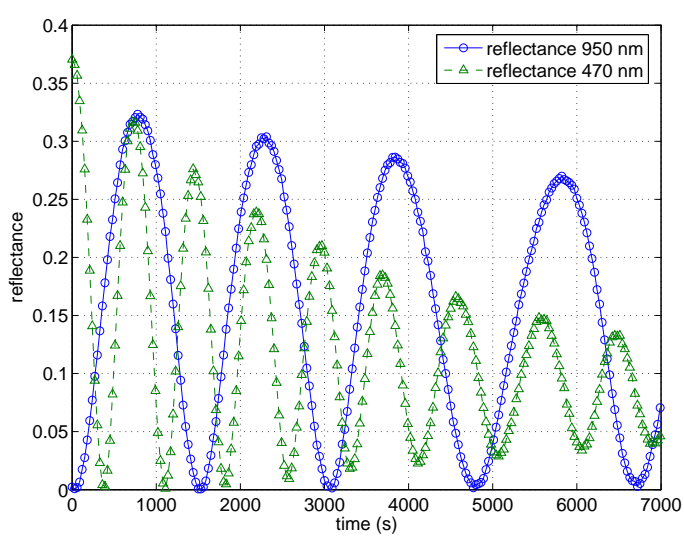

Fig. 6. Simulated reflectance at $950 \mathrm{~nm}$ and $470 \mathrm{~nm}$ based on exponential decay of film growth rate and linearly increasing roughness.

TABLE I

SiMULATION PARAMETERS.

\begin{tabular}{|c|c|c|c|c|c|}
\hline & \multicolumn{2}{|c|}{$\overline{Q_{k}}$} & \multicolumn{3}{|c|}{$\hat{\mathbf{x}}_{0}$} \\
\hline Case I & $\begin{array}{c}0.1 \\
0\end{array}$ & $\begin{array}{c}0 \\
0.1\end{array}$ & {$[120$} & 10 & $10]^{T}$ \\
\hline Case II & $\begin{array}{c}100 \\
0\end{array}$ & $\begin{array}{c}0 \\
100\end{array}$ & {$[120$} & 10 & $10]^{T}$ \\
\hline Case III & $\begin{array}{c}100 \\
0\end{array}$ & $\begin{array}{c}0 \\
100\end{array}$ & {$[120$} & 5 & $5]^{T}$ \\
\hline Case IV & $\begin{array}{c}100 \\
0\end{array}$ & $\begin{array}{c}0 \\
100\end{array}$ & {$[110$} & 5 & $5]^{T}$ \\
\hline
\end{tabular}

to be random constant variables so that EKF can estimate them adaptively. The covariance matrix of process model disturbances, $Q_{k}$, essentially specifies the range that EKF could adjust these random variables. Larger $Q_{k}$ gave EKF more flexibility to adjust roughness and growth rate and thus made it more possible to capture the trend of roughness and growth rate change. Therefore when applying EKF to real experimental data, $Q_{k}$ should be tuned higher to get better performance in the presence of an uncertain process model.

In Case III of Table I, the effect of the initial state estimate on EKF performance was studied. Generally speaking, discrepancy in the initial state estimate could cause error in later estimates. In the extended Kalman filter the new estimate was calculated by linearizing the process and sensor models at the previous state estimate. Therefore erroneous initial estimates can propagate into later estimates. The EKF had different sensitivities to different state variables. Figure 11 shows the simulation results when the EKF used the larger $Q_{k}$ in Table I and an erroneous initial state estimate (Case III). The initial film thickness estimate is correct but both roughness and film growth rate have an error of 50\%. As shown, the EKF still yielded a satisfactory estimate. However, using the initial estimate of Case IV in Table I, which used an initially correct roughness and growth rate estimate, but only a $10 \%$ error in thickness,
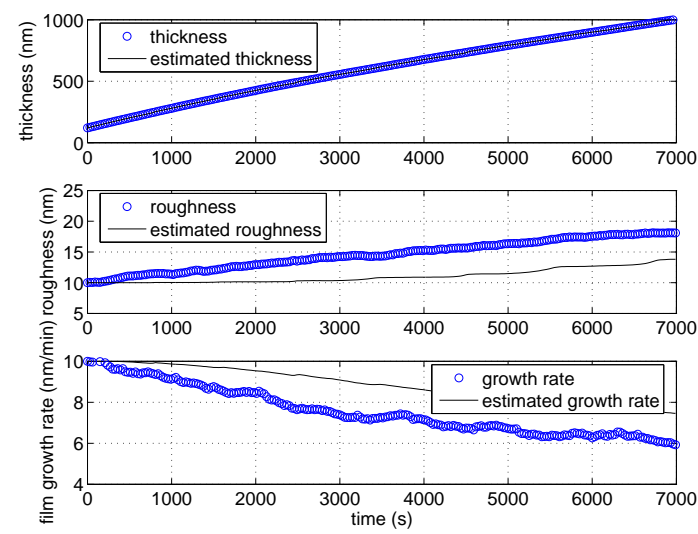

Fig. 7. Comparison of estimated and actual states for Case I of Table I.

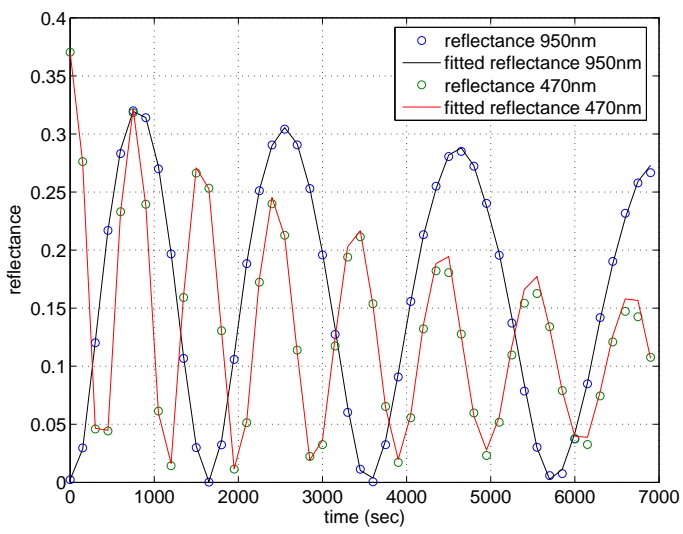

Fig. 8. Comparison of estimated and actual reflectance for Case I of Table I.
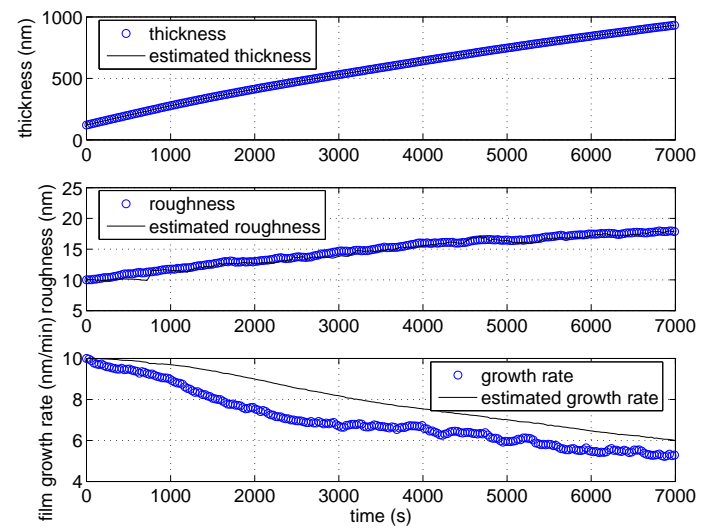

Fig. 9. Comparison of estimated and actual states for Case II of Table I. 


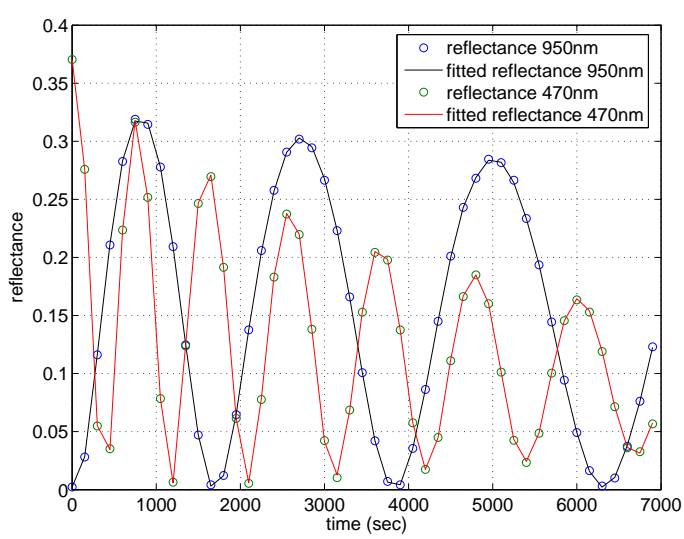

Fig. 10. Comparison of estimated and actual reflectance for Case II of Table I.
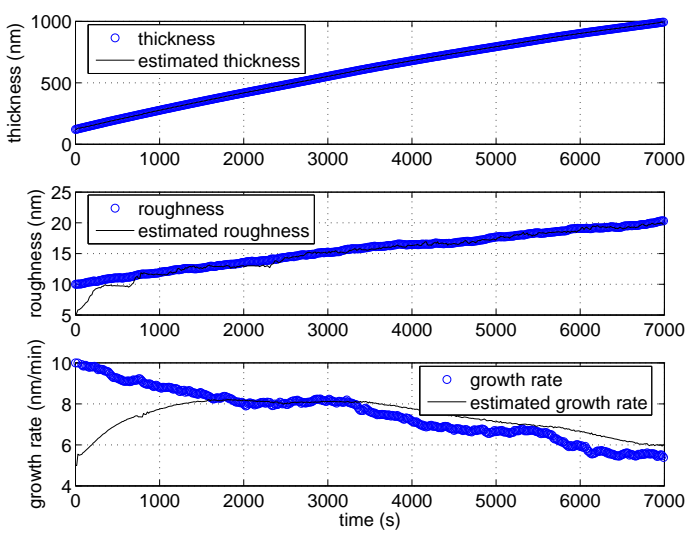

Fig. 11. Comparison of estimated and actual states for Case III in Table I.

the EKF cannot yield a satisfactory estimate, as shown in Figure 12. The oscillations in reflectance are strongly correlated with the film thickness, and a small error in initial film thickness can lead to a large discrepancy between the measured and estimated reflectance. Once the estimate gets ahead or behind by one oscillation, we observe that the EKF is not able to correct for this error due to the nonlinear nature of the reflectance oscillations.

\section{EXPERIMENTAL}

\section{A. Chemical vapor deposition reactor}

Yttria-stabilized zirconia (YSZ) deposited by metalorganic chemical vapor deposition (MOCVD) was used to demonstrate the feasibility of the EKF based soft sensor. This material system was selected for its utility in demonstrating concepts, but is also of interest in a wide range of applications [48], [49], [50]. Figure 13 shows a photograph of the apparatus. A schematic is shown in Figure 14. It mainly consisted of three parts: material supply system including $\mathrm{Ar}$ and $\mathrm{O}_{2}$ gas supply and two precursor evaporators, a deposition chamber with in-situ reflectometer/pyrometer, and a vacuum system with automatic pressure control. The $\beta$-diketonates $\mathrm{Zr}(\mathrm{tmhd})_{4}$ and $\mathrm{Y}(\mathrm{tmhd})_{3}$
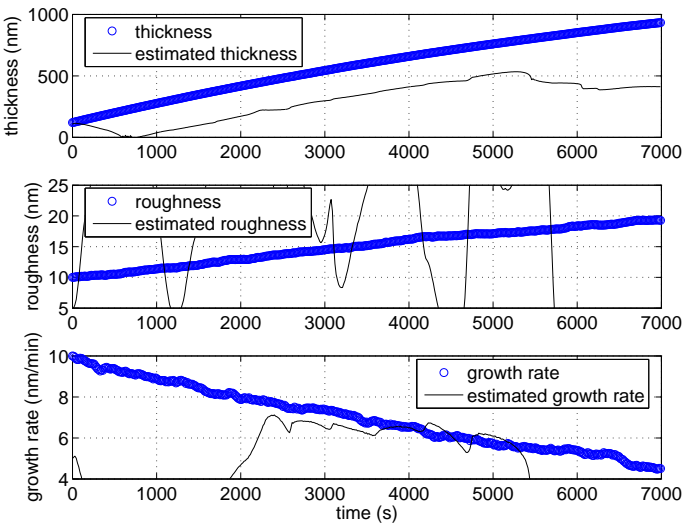

Fig. 12. Comparison of estimated and actual states for Case IV in Table I.

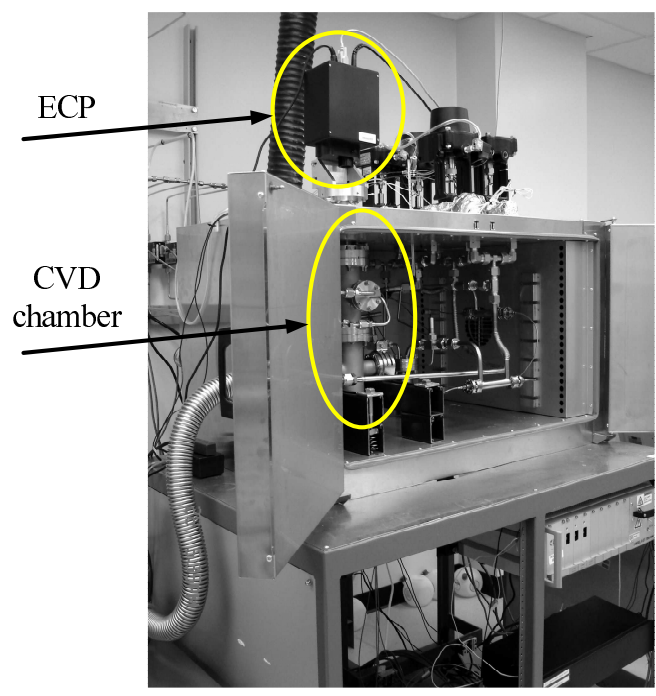

Fig. 13. Photograph of the CVD reactor.

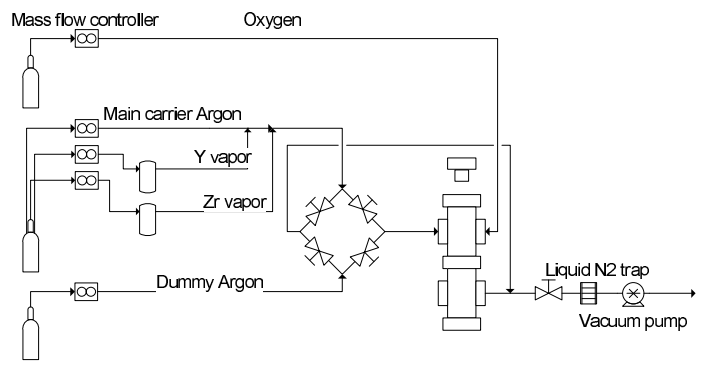

Fig. 14. Reactor schematic. 
[Strem Chemicals, Inc.] were used as the precursors due to their high volatility at temperature below $250^{\circ} \mathrm{C}$ and their thermal stability in the gas phase at elevated temperatures [51]. The precursors were evaporated in separate stainlesssteel evaporators and carried to a flow manifold via $\mathrm{Ar}$ gas. Another stream of Ar with the same flow rate as the Ar carrying precursors entered the manifold from the other side. The manifold consisted of four bellows-sealed valves (two normally closed and two normally open) from Swagelok. These valves were controlled by solenoid valves thus allowing us to either flow precursors over the reactor to deposit, or to bypass the reactor and flow pure Ar over the substrate during heat up and cool down steps of the processing. Oxygen was piped through a separate tube and mixed with the precursor stream as it entered the deposition chamber, since premature reactions between the precursors and oxygen can lead to undesired gas-phase particle formation. Another Ar stream with relatively slower flow rate was used as purge flow to prevent precursor condensation on the sensor viewport. Pipes carrying precursor vapor were kept at a temperature slightly higher than the evaporation temperature in order to suppress condensation. All gas flow rates were controlled by mass flow controllers from MKS. The vacuum system was made up of a vacuum pump, a pressure transducer, a pressure controller and a throttle valve from MKS. The system was designed to operate at a pressure range of $1-10$ torr which is common for most MOCVD processes. Before the vacuum pump a liquid nitrogen trap was used to capture escaped precursors.

The deposition chamber consists of two standard stainless-steel ultra-high vacuum (UHV) reducing crosses, with a chamber diameter of 2 ". The growth chamber was enclosed in an oven to prevent precursor condensation on the chamber walls. A 1" diameter resistive sample heater with a substrate block designed for oxidizing conditions, both supports and heats the substrate. A vertical flow geometry was selected for the MOCVD reactor. This geometry is typically used in cold wall designs for rapid thermal processing, with cold walls creating a temperature gradient and the need for temperature control. Another reason for the popularity of the vertical flow geometry is the existence of a one-dimensional similarity solution in the limit of an infinite wafer.

The in-situ sensor used in this study is actually an emissivity-correcting pyrometer. This unit [from SVT Associates] contains all the optics and electronics, and requires only a single normal-incidence viewport. The pyrometer measures the emission at $950 \mathrm{~nm}$ and $850 \mathrm{~nm}$, and the LED reflectometer measures normal reflectance at $950 \mathrm{~nm}$ and $470 \mathrm{~nm}$. Its sampling time is $1 \mathrm{sec}$. This system was designed to be used with semiconductors that are opaque at $950 \mathrm{~nm}$ which is the case for silicon substrate. In this study, only the reflectometer data were processed. By incorporating pyrometer data into the estimator, more information could be interpreted. This work is currently underway.
TABLE II

DEPOSITION CONDITIONS.

\begin{tabular}{|l|r|}
\hline Evaporation temperature: $\mathrm{Z}(\mathrm{tmhd})_{4}\left({ }^{\circ} \mathrm{C}\right)$ & 205 \\
Evaporation temperature: $\mathrm{Y}(\mathrm{tmhd})_{3}\left({ }^{\circ} \mathrm{C}\right)$ & 125 \\
Deposition temperature $\left({ }^{\circ} \mathrm{C}\right)$ & 700 \\
Total pressure (torr) & $2-6$ \\
Deposition time $(\mathrm{min})$ & 240 \\
Molar ratio of $\mathrm{O}_{2}$ & $25 \%$ \\
Total gas flow rate $(\mathrm{sccm})$ & 500 \\
\hline
\end{tabular}

The reactor was operated using a custom LabView program, which also acquires experimental data. The reactor was operated under conditions similar to those previously reported in the literature [52]—-typical experimental conditions were listed in Table II. Films were deposited on 1" (100) Si wafers with one side polished obtained from Nova Electronic Materials, Inc, and were cleaned with organic solvents.

Ex-situ characterization of the YSZ films was performed to validate the estimated film properties. Film thickness was measured with a M-2000 ellipsometer (J. A. Woollam Co., Inc.). Surface roughness was measured using a PicoPlus AFM (Molecular Imaging). Ellipsometry measurements of the YSZ film were not well fit with a three-phase model (air, YSZ, silicon), suggesting that the film may be rough or nonuniform over the large spot size of the ellipsometer. However, the number of oscillations in the data indicated a thickness in the range of 600 to $700 \mathrm{~nm}$. AFM images before and after growth indicated that the initial silicon surface has an rms roughness of $10 \mathrm{~nm}$, while after growth the surface roughness is $65 \mathrm{~nm}$.

\section{EXPERIMENTAL RESULTS}

\section{A. Raw data preprocessing}

When applying EKF to real experimental data, the reflectometer must be calibrated first so that the raw voltage signal acquired by the sensor can be transformed to reflectance that the EKF can use. The reflectometer measures the intensity of the light reflected back from the surface and captured by the detector. Therefore reflectance is a linear function of voltage. The linearity depends on the reactor geometry, relative position and optical aperture of the sensor. The reflectometer was first calibrated at room temperature with a zero reflectance case and a known reflectance of a substrate. The zero reflectance case was performed with the substrate missing, and represents a baseline measurement for which stray light is accounted for in the calibration. A known substrate reflectance was obtained by using a bare silicon wafer. The normal reflectance of silicon at room temperature can be calculated from its refractive index.

This sensor calibration was performed at room temperature. However when the silicon wafer was heated up to reaction temperature (e.g. $700^{\circ} \mathrm{C}$ ), erroneous reflectance readings were observed. Figure 15 shows a typical measured reflectance data when YSZ was deposited on a silicon wafer at $700^{\circ} \mathrm{C}$. The oscillation after around $8000 \mathrm{~s}$ was 


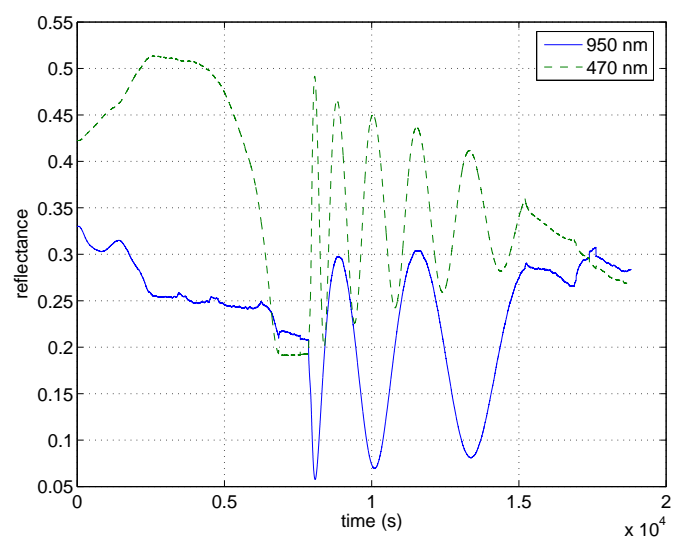

Fig. 15. Reflectance vs. time during heating up of the wafer (before $8000 \mathrm{~s}$ ) and during film growth (after $8000 \mathrm{~s}$ ).

caused by the film growth. The data before $8000 \mathrm{~s}$ are measured reflectance when the wafer was heated up from room temperature to the reaction temperature. As shown, reflectance at $470 \mathrm{~nm}$ decreased gradually while reflectance at $950 \mathrm{~nm}$ increased first (up to $3000 \mathrm{~s}$ ) and then decreased sharply. The change of reflectance during heatup cannot be explained the temperature dependence of the optical constants of silicon. According to Jellison, refractive index of silicon increases with temperature for all light wavelengths longer than $365 \mathrm{~nm}$ [43]. An increasing refractive index should cause the increase of reflectance. The reflectance change in Fig. 15 is also not repeatable. However in all experiments, after reaction temperature was stabilized, reflectance also stabilized as shown in Fig. 15 (after around $7000 \mathrm{~s}$ ). This led us to believe that the linear relation obtained at room temperature calibration may have changed when the wafer was heated up due to thermal expansion and shifting alignment.

If the erroneous reflectance data was fed to EKF directly, our preliminary results indicated that the EKF would fail because the mismatch between the measured and predicted reflectance was too large to handle. Adding additional fitting parameters to compensate for the sensor model deficiency was found to be helpful [12], but by doing so, information embedded in the measurement, such as amplitude decay in Fig. 15, can not be extracted. The method used in this study was to calibrate the reflectometer at high temperature after reaction temperature was achieved, and the reflectance measurement stabilized. The reflectance of bare silicon at reaction temperature (e.g. $700^{\circ} \mathrm{C}$ ) could be used as the first point for calibration because its reflectance could be calculated from its refractive index at that temperature. The second point for calibration was chosen to be the first peak of reflectance at $470 \mathrm{~nm}$. The reflectance at this point was calculated from the sensor model eq. (5). The data preprocessing could also obtain initial film thickness and growth rate estimates. The thickness was calculated from the half wavelength in the film. Thickness divided by the time gives an estimate of film growth rate. A disadvantage
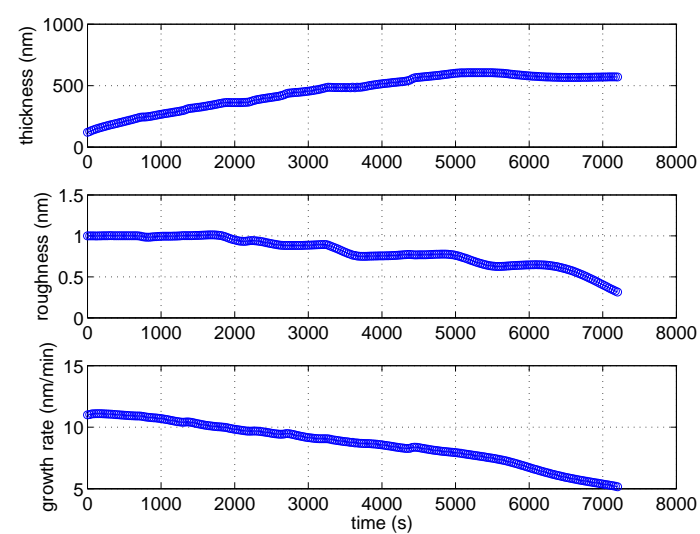

Fig. 16. Estimated film properties from preprocessed reflectance data.

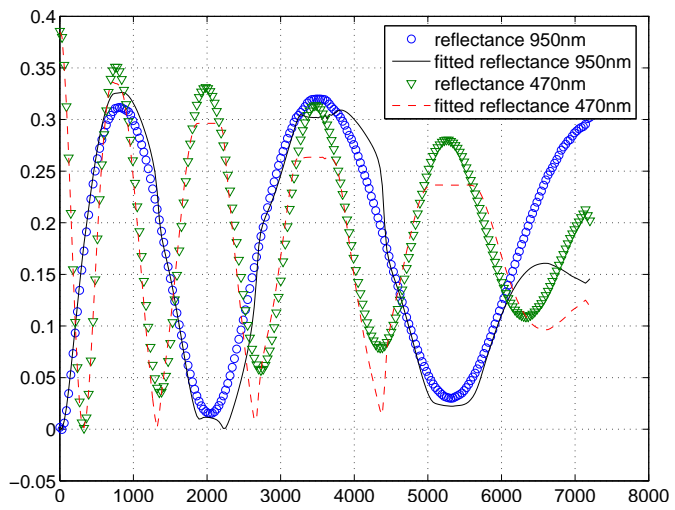

Fig. 17. Model predicted reflectance and measured reflectance.

with this data preprocessing method is that the data before the first peak appeared is not estimated.

\section{B. Application of EKF for in-situ sensing YSZ}

When applying EKF to preprocessed reflectance data, the understanding of the behavior of EKF obtained in the simulation study proved to be quite helpful. Generally speaking $Q_{k}$ should be set large enough for EKF to adjust the random state variables adaptively. The initial estimate of film thickness should be as accurate as possible. The thickness at that point was calculated to be $120.8 \mathrm{~nm}$ based on the refractive index of the yttria film at the growth temperature. The average film growth rate from start of the reaction to the first peak at $470 \mathrm{~nm}$ was calculated to be around $11 \mathrm{~nm} / \mathrm{min}$. The silicon wafer roughness after heating up to reaction temperature and then cooling down was ex-situ characterized with AFM and found to be in the range of 20 to $30 \mathrm{~nm}$. This roughness is actually that of the interface of $\mathrm{SiO}_{2}$ on top of the silicon substrate. The surface roughness at the first peak is not known but assumed to be the same as that of the silicon substrate, so an initial state estimate of $\left[\begin{array}{lll}120.8 & 1 & 11\end{array}\right]^{T}$ was used. The covariance matrix of sensor noise was chosen to be the same as in the simulation study. The estimated states are shown in Fig. 16. Figure 17 compares the predicted 
reflectance with measured data. As shown in Fig. 16, the film growth rate was estimated to decrease from $11 \mathrm{~nm} / \mathrm{min}$ to $5 \mathrm{~nm} / \mathrm{min}$. As discussed in the sensor model simulation section, film growth rate determines the period of oscillation. The measured reflectance in Fig. 17 indicated an increasing oscillation period which is consistent with the estimated growth rate decay. Figure 16 also shows the estimated thickness to be $535 \mathrm{~nm}$. The monotonic increase of the thickness indicated that a simple process model helped EKF to determine the direction of thickness evolution. The film thickness increased nonlinearly until the end of the experiment where it nearly stopped increasing. Again this is consistent with the estimate of growth rate decay. The estimated film roughness did not change significantly from the initial estimate. When we used a different initial estimate of film roughness, the new estimated roughness did not change either and was always close to the initial estimate. As discussed in the sensor model simulation section, increasing surface roughness would cause the peak value of reflectance to decay but does not affect valley reflectance very much. The reflectance data in Fig. 17 did not have this kind of feature so EKF had no necessary information to adjust the roughness. The data suggests that the roughness is not changing significantly over the growth period, although the roughness at the initial calibration point may in fact be nonzero. Further ex-situ characterization and validation of the sensor model is need, but the preliminary experimental results show that inclusion of a process model can aid in the interpretation of the sensor data to infer thickness and roughness.

\section{SUMMARY AND CONCLUSIONS}

An extended Kalman filter was used to estimate film thickness, roughness, and growth rate during chemical vapor deposition from reflectance measurements at two wavelengths. A sensor model was derived to incorporate the effect of roughness and refractive index on surface reflection, and a simple process model was used to specify the direction of thickness evolution. The EKF captured the trend of film growth decay and overcame the problem of local minima encountered in the least square fitting method. Preliminary results suggest that the EKF or other systematic estimation techniques could be a viable approach for estimating film properties in-situ during deposition.

The application of feedback control in chemical vapor deposition is challenging, because the sensors do not provide direct measurements of the properties to be controlled, and because the systems are batch and nonlinear. For both reasons, a good understanding of the process dynamics is needed to develop a model to interpret the sensor data. In many cases the required model is multiscale. Discrete models describing individual atomic interactions or the velocity profiles of discrete grains may be needed to describe the process dynamics [53], [54], [55]. However, a detailed model also has disadvantages in process control. It reduces the applicability of the controller, and the time required to build the model may not be cost-effective. Additionally, it may have a large number of parameters that need to be estimated periodically to account for equipment drift. Also, fast computation is required for online implementation. Ongoing research in multiscale modeling may reduce the computation enabling online implementation, with approaches including equation-free computing [56], adaptive tabulation [57], or reduced-order modeling [58]. In addressing problems in sensing in CVD, this tradeoff must be continually assessed to reduce model complexity while retaining the dynamics of interest.

\section{ACKNOWLEDGMENTS}

This work was supported by an NSF CAREER grant A Systems Approach to Materials Processing, and by the Georgia Tech Foundation.

\section{REFERENCES}

[1] A. A. R. Elshabini-Riad and F. D. Barlow, Thin film technology handbook, ser. Electronic packaging and interconnection series. New York: McGraw-Hill, 1998.

[2] S. A. Campbell, The science and engineering of microelectronic fabrication. New York: Oxford University Press, 1996.

[3] A. R. Boccaccini and I. Zhitomirsky, "Application of electrophoretic and electrolytic deposition techniques in ceramics processing," Current Opinion In Solid State \& Materials Science, vol. 6, no. 3, pp. 251-260, 2002.

[4] K. L. Choy, "Chemical vapour deposition of coatings," Progress In Materials Science, vol. 48, no. 2, pp. 57-170, 2003.

[5] A. Goetzberger, C. Hebling, and H. W. Schock, "Photovoltaic materials, history, status and outlook," Materials Science \& Engineering $R$-Reports, vol. 40, no. 1, pp. 1-46, 2003.

[6] H. O. Pierson and H. O. Pierson, Handbook of chemical vapor depostion [i.e. deposition] (CVD): principles, technology, and applications, 2nd ed. Norwich, N.Y.: Noyes Publications/William Andrew Pub., 1999.

[7] T. F. Edgar, S. W. Butler, W. J. Campbell, C. Pfeiffer, C. Bode, S. B. Hwang, K. S. Balakrishnan, and J. Hahn, "Automatic control in microelectronics manufacturing: Practices, challenges, and possibilities," Automatica, vol. 36, no. 11, pp. 1567-1603, 2000.

[8] J. Moyne, E. del Castillo, and A. M. Hurwitz, Run-to-run control in semiconductor manufacturing. Boca Raton, FL: CRC Press, 2001.

[9] Y. M. Lou and P. D. Christofides, "Feedback control of surface roughness of GaAs (001) thin films using kinetic Monte Carlo models," Computers \& Chemical Engineering, vol. 29, no. 1, pp. 225-241, 2004.

[10] _ - "Feedback control of surface roughness in sputtering processes using the stochastic Kuramoto-Sivashinsky equation," Computers \& Chemical Engineering, vol. 29, no. 4, pp. 741-759, 2005.

[11] D. E. Aspnes, W. E. Quinn, and S. Gregory, "Optical Control Of Growth Of Alxga1-Xas By Organometallic Molecular-Beam Epitaxy," Applied Physics Letters, vol. 57, no. 25, pp. 2707-2709, 1990.

[12] T. L. Vincent and P. P. Khargonekar, "An extended Kalman filter based method for fast in-situ etch rate measurements," in Materials Research Society Symposium Proceedings, vol. 406, 1996, pp. 87-93.

[13] Y. Inoue, H. Sugimura, and O. Takai, "In situ observation of behavior of organosilicon molecules in low-temperature plasma enhanced CVD," Thin Solid Films, vol. 345, no. 1, pp. 90-93, 1999.

[14] S. Katai, A. Kovats, I. Maros, and P. Deak, "Ion energy distributions and their evolution during bias-enhanced nucleation of chemical vapor deposition of diamond," Diamond And Related Materials, vol. 9, no. 3-6, pp. 317-321, 2000.

[15] M. Mermoux, L. Fayette, B. Marcus, N. Rosman, L. Abello, and G. Lucazeau, "In situ analysis of the Raman diamond line. Measurements in the visible and UV spectral range," Physica Status Solidi A-Applied Research, vol. 154, no. 1, pp. 55-68, 1996.

[16] I. Schmidt, F. Hentschel, and C. Benndorf, "Low temperature diamond growth using fluorinated hydrocarbons," Diamond And Related Materials, vol. 5, no. 11, pp. 1318-1322, 1996. 
[17] J. Rodriguezviejo, F. Sibieude, and M. T. Clavagueramora, "HighTemperature Oxidation Of Cvd Beta-Sic.1. Experimental-Study," Journal Of The European Ceramic Society, vol. 13, no. 2, pp. 167175,1994

[18] G. Ruhl, R. Rehmet, M. Knizova, R. Merica, and S. Veprek, "In situ XPS studies of the deposition of TiNxCy films from tetrakis(dimethylamido)titanium(IV) and bis[N,N'-bis(tertbutyl)ethylenediamido]titanium(IV)," Chemistry Of Materials, vol. 8, no. 12, pp. 2712-2720, 1996.

[19] M. Tabbal, R. Izquierdo, M. Meunier, C. Pepin, and A. Yelon, "Surface characterization of excimer laser induced deposition of W on GaAs from WF6 and H-2," Applied Surface Science, vol. 108, no. 4, pp. 417-424, 1997.

[20] A. J. Springthorpe, T. P. Humphreys, A. Majeed, and W. T. Moore, "Insitu Growth-Rate Measurements During Molecular-Beam Epitaxy Using An Optical-Pyrometer," Applied Physics Letters, vol. 55, no. 20, pp. 2138-2140, 1989.

[21] H. Grothe and F. G. Boebel, "Insitu Control Of Ga(Al)As Mbe Layers By Pyrometric Interferometry," Journal Of Crystal Growth, vol. 127, no. 1-4, pp. 1010-1013, 1993.

[22] I. An, Y. M. Li, C. R. Wronski, H. V. Nguyen, and R. W. Collins, "Insitu Determination Of Dielectric Functions And Optical Gap Of Ultrathin Amorphous-Silicon By Real-Time Spectroscopic Ellipsometry," Applied Physics Letters, vol. 59, no. 20, pp. 2543-2545, 1991.

[23] D. E. Aspnes, W. E. Quinn, M. C. Tamargo, M. A. A. Pudensi, S. A. Schwarz, M. Brasil, R. E. Nahory, and S. Gregory, "Growth Of Alxga1-Xas Parabolic Quantum-Wells By Real-Time FeedbackControl Of Composition," Applied Physics Letters, vol. 60, no. 10, pp. 1244-1246, 1992.

[24] R. Droopad, C. H. Kuo, S. Anand, K. Y. Choi, and G. N. Maracas, "Determination Of Molecular-Beam Epitaxial-Growth Parameters By Ellipsometry," Journal Of Vacuum Science \& Technology B, vol. 12, no. 2, pp. 1211-1213, 1994.

[25] C. H. Kuo, S. Anand, R. Droopad, K. Y. Choi, and G. N. Maracas, "Measurement Of Gaas Temperature-Dependent Optical-Constants By Spectroscopic Ellipsometry," Journal Of Vacuum Science \& Technology B, vol. 12, no. 2, pp. 1214-1216, 1994.

[26] Y. Z. Hu, D. J. Diehl, Q. Liu, C. Y. Zhao, and E. A. Irene, "In-Situ Real-Time Measurement Of The Incubation-Time For Silicon Nucleation On Silicon Dioxide In A Rapid Thermal-Process," Applied Physics Letters, vol. 66, no. 6, pp. 700-702, 1995.

[27] C. D. Zuiker, D. M. Gruen, and A. R. Krauss, "Laser-Reflectance Interferometry Measurements Of Diamond-Film Growth," Mrs Bulletin, vol. 20, no. 5, pp. 29-31, 1995.

[28] _ _ "In situ laser reflectance interferometry measurement of diamond film growth," Journal Of Applied Physics, vol. 79, no. 7, pp. 3541-3547, 1996.

[29] W. G. Breiland and K. P. Killeen, "A Virtual Interface Method For Extracting Growth-Rates And High-Temperature Optical-Constants From Thin Semiconductor-Films Using In-Situ Normal Incidence Reflectance," Journal Of Applied Physics, vol. 78, no. 11, pp. 67266736, 1995.

[30] D. E. Aspnes, "Minimal-Data Approaches For Determining OuterLayer Dielectric Responses Of Films From Kinetic Reflectometric And Ellipsometric Measurements," Journal Of The Optical Society Of America A-Optics Image Science And Vision, vol. 10, no. 5, pp. 974-983, 1993.

[31] C. K. Chui and G. Chen, Kalman Filtering with Real-Time Applications. Berlin: Springer, 1999.

[32] P. S. Maybeck, Stochastic Models, Estimation and Control. New York: Academic Press, 1979

[33] P. Atkinson and M. Nixon, "Monitoring a deposition process using an extended Kalman filter," IEE Porceddings-D Control Theory and Applications, vol. 131, no. 1, pp. 1-7, 1984.

[34] W. W. Woo, S. A. Avoronos, H. O. Sankur, J. Bajaj, and S. J. C. Irvine, "In-situ estimation of MOCVD growth rate via a modified Kalman filter," AIChE Journal, vol. 1996, p. 1319, 1996.

[35] T. L. Vincent, P. P. Kargonekar, and F. L. Terry, "End point and etch rate control using dual wavelength laser reflectometry with a nonlinear estimator,' J. of Electrochemical Society, vol. 144, p. 2467, 1997.

[36] K. Torrance and E. Sparrow, "Theory for off-specular reflection from roughed surfaces," Journal of the Optical Society of America, vol. 57, pp. 1105-1114, 1967.
[37] K. Tang, Y. Yang, and R. O. Buckius, "Theory and experiments on scattering from rough interfaces," Annaul Review of Heat Transfer, vol. 10, pp. 100-140, 1999.

[38] I. Filinski, "Effects Of Sample Imperfections On Optical-Spectra," Physica Status Solidi B-Basic Research, vol. 49, no. 2, pp. 577-\&, 1972.

[39] Z. Yin, H. S. Tan, and F. W. Smith, "Determination of the optical constants of diamond films with a rough growth surface," Diamond And Related Materials, vol. 5, no. 12, pp. 1490-1496, 1996.

[40] X. F. Xu and C. P. Grigoropoulos, "High-Temperature Radiative Properties Of Thin Polysilicon Films At The Lambda $=0.6328 \mathrm{Mu}-$ M Wavelength," International Journal Of Heat And Mass Transfer, vol. 36, no. 17, pp. 4163-4172, 1993.

[41] Y. Akiyama, N. Imaishi, Y. S. Shin, and S. C. Jung, "Macro- and micro-scale simulation of growth rate and composition in MOCVD of yttria-stabilized zirconia," Journal of Crystal Growth, vol. 241, no. 3, pp. 352-362, 2002.

[42] C. K. Chui and G. Chen, Kalman filtering: with real-time applications, 3rd ed. Berlin; New York: Springer, 1999.

[43] G. E. Jellison, "Optical functions of silicon at elevated-temperatures," Journal of Applied Physics, vol. 76, no. 6, p. 3758, 1994.

[44] D. L. Wood, K. Nassau, and T. Y. Kometani, "Refractive-index of Y2O3 stabilized cubic zirconia-variation with composition and wavelength," Applied Optics, vol. 29, no. 16, pp. 2485-2488, 1990.

[45] E. D. Palik, Ed., Handbook of the Optical Constants of Solids II. Boston: Academic Press, 1991

[46] J. L. Luo, "Study on the growth of CVD diamond thin films by in situ reflectivity measurement," Diamond and Related Materials, vol. 11, no. 11, pp. 1871-1875, 2002.

[47] W. G. Breiland and K. P. Killeen, "A virtual interface method for extracting growth-rates and high-temperature optical-constants from thin semiconductor-films using in-situ normal incidence reflectance," Journal of Applied Physics, vol. 78, p. 6726, 1995.

[48] M. Sayer and K. Sreenivas, "Ceramic thin-films," Science, vol. 247, pp. 1056-1060, 1990

[49] A. Asada, "Limiting current type of oxygen sensor with highperformance," Sensors and Actuators B, vol. 1, pp. 312-318, 1990.

[50] J. T. Brown, "Solid oxide fuel cells," IEEE Transactions on Energy Conversion, vol. 3, pp. 193-198, 1988.

[51] M. Pulver, "Deposition of $\mathrm{ZrO} 2$ and $\mathrm{Y} 2 \mathrm{O} 3$-stabilized $\mathrm{ZrO} 2$ from beta-diketonates," Journal De Physique IV, vol. 3, pp. 305-312, 1993.

[52] Y. Akiyama, N. Imaishi, Y.-S. Shin, and S.-C. Jung, "Macro- and micro-scale simulation of growth rate and composition in MOCVD of yttria-stabilized zirconia," Journal of Crystal Growth, vol. 241, pp. 352-362, 2002.

[53] H. Huang and G. H. Gilmer, "Texture competition during thin film deposition-effects of grain boundary migration," Computational Materials Science, vol. 23, pp. 190-196, 2002.

[54] P. Vogl, U. Hansen, and V. Fiorentini, "Multiscale approaches for metal thin film growth," Computational Materials Science, vol. 24, pp. 58-65, 2002.

[55] R. M. Nieminen, "From atomistic simulation toward multiscale modeling of materials," Journal of Physics: Condensed Matter, vol. 14, pp. 2859-2876, 2002.

[56] C. W. Gear, I. G. Kevrekidis, and C. Theodoropoulos, "'Coarse' integration/bifurcation analysis via microscopic simulators: microGalerkin methods," Computers and Chemical Engineering, vol. 26, no. 7-8, pp. 941-963, 2002.

[57] J. D. Hedengren and T. F. Edgar, "In situ adaptive tabulation for realtime control," Industrial \& Engineering Chemistry Research, vol. 44, no. 8 , p. $2716,2005$.

[58] M. A. Gallivan and R. M. Murray, "Reduction and identification methods for markovian control systems, with application to thin film deposition," International Journal of Robust and Nonlinear Control, vol. 14, no. 2, pp. 113-132, 2004. 